\title{
O processo de detecção e tratamento de casos de tuberculose em um presídio
}

\author{
The process of detection and treatment of cases of tuberculosis \\ in a prison
}

\author{
Mariana Soares Valença ${ }^{1}$ \\ Marta Regina Cezar-Vaz ${ }^{2}$ \\ Clarice Brinck Brum ${ }^{3}$ \\ Pedro Eduardo Almeida da Silva ${ }^{3}$
}

${ }^{1}$ Centro de Ciências da Vida e da Saúde, Universidade Católica de Pelotas.

R. Gonçalves Chaves 373, Centro. 96015-560 Pelotas RS Brasil. mariana. valenca@ucpel.edu.br 2 Escola de Enfermagem, Universidade Federal do Rio Grande (FURG). Rio Grande RS Brasil.

${ }^{3}$ Faculdade de Medicina, FURG. Rio Grande RS Brasil.

\begin{abstract}
This study seeks to analyze the process of detection and treatment of cases of tuberculosis (TB) in a prison in the south of Brazil. An active and passive search for TB was conducted to estimate the scale of TB in a prison with 764 inmates. In conjunction with the detection strategies and clinical follow-up of the $41 \mathrm{~TB}$ cases, participant observation and records in field diaries were performed, making it possible to analyze the scope and limitations of detection and treatment of cases of $T B$ in prison. The development of search strategies is discussed along with the use of questionnaires to detect symptomatic cases, as well as the inadequacy of the clinical follow-up of TB cases, involvement of different workers and coordination between prison and health services. There is clear potential for the control of TB using an active search to induce the passive detection and screening for symptoms that - even skewed by the perceptions of inmates regarding symptoms of $T B$ - enabled an increase in detection. The functional dynamics of prison life hamper the inclusion of health routines and can restrict actions to control TB and other diseases. In the process of control of TB in prisons, the feasibility of effective detection methods is as important as planning based on disease conditions, network services and workers involved.
\end{abstract}

Key words Tuberculosis, Prisons, Diagnosis, Treatment
Resumo $O$ estudo objetiva analisar o processo de detecção e tratamento de casos de tuberculose (TB) em um presídio do sul do Brasil. Realizou-se busca ativa e passiva para estimar a magnitude da TB entre uma população de 764 detentos. Simultaneamente às estratégias de detecção e ao seguimento clínico dos 41 casos confirmados, realizouse observação participante e registros em diário de campo, o que possibilitou analisar potenciais e limitações das ações de detecção e tratamento da $T B$ prisional. Discute-se o desenvolvimento das estratégias de busca, o uso de questionários para detecção de sintomáticos, a fragilidade do seguimento clínico dos casos de TB, o envolvimento de diferentes trabalhadores e a articulação entre presídio e serviços de saúde. São potencialidades para o controle da TB utilizar a busca ativa como indutora da detecção passiva e a triagem por sintomas que, mesmo interferida pelas percepções dos detentos sobre os sintomas de TB, permitiu aumentar a detecção. A dinâmica funcional do presídio dificulta a inserção de rotinas de saúde, podendo limitar ações de controle da TB e outras enfermidades. No processo de controle da TB em presídios, a exequibilidade de métodos de detecção eficazes é tão importante quanto planejá-lo a partir das condições de adoecimento, rede de serviços e trabalhadores envolvidos.

Palavras-chave Tuberculose, Prisões, Diagnóstico, Tratamento 


\section{Introdução}

O controle da tuberculose (TB) nas instituições penais desempenha condição crítica na carga da doença de maneira global. A prevalência mundial de TB estimada em 2013 foi 159/100.000', nos presídios relata-se uma prevalência média de $1.913 / 100.000^{2}$. Nas prisões brasileiras, a incidência de TB reportada em 2013 foi 1.080/100.000, índice 31 vezes superior ao notificado entre a população livre, $35 / 100.000^{3}$.

Entre os fatores associados à $\mathrm{TB}$ em presídio destacam-se, a origem de comunidades socioeconomicamente desfavorecidas, a baixa escolaridade, a TB prévia, os antecedentes de encarceramento e o HIV ${ }^{4-6}$.

Sugere-se que as limitações para o controle da doença em presídios estejam atreladas a imprecisão de algoritmos diagnósticos e a falta de estrutura laboratorial adequada. Sendo a recomendação principal aumentar a frequência das triagens de casos com suspeita de $\mathrm{TB}^{2}$.

Os presídios são ambientes repletos de particularidades que podem interferir no desenvolvimento de ações voltadas à saúde ${ }^{7}$, entretanto, os aspectos operacionais do controle da $\mathrm{TB}$ em presídios não têm sido amplamente abordados pela literatura científica. Portanto, este estudo tem como objetivo, analisar o processo de detecção e tratamento de casos de TB em um presídio da região Sul do Brasil.

\section{Métodos}

Partindo do pressuposto de que a complementaridade de estudos quantitativos e qualitativos propulsione novos insights e um maior entendimento dos problemas de pesquisa da área da saúde para atingir o objetivo proposto, utilizaram-se métodos mistos 8 . Para possibilitar interpretações sobre os potenciais e as limitações do processo detecção e tratamento de casos de TB em um presídio, a abordagem quantitativa descritiva do tipo inquérito foi desenvolvida concomitantemente à abordagem qualitativa descritiva exploratória.

O estudo foi realizado sob condições de pesquisa em um presídio do sul do Brasil que, na época, abrigava 764 detentos. No período de 12 meses (novembro de 2012-13), foram desenvolvidas ações de busca ativa e passiva para detecção de casos de TB pulmonar.

Os participantes da busca ativa foram selecionados aleatoriamente e compuseram amostra representativa da população do presídio. Para tanto, o cálculo da amostra utilizou o total de 764 presos, com uma prevalência de TB de $10 \%{ }^{6,9}$ e um nível de confiança de $95 \%$, com um erro amostral de $+/-5 \%$, totalizando 285 detentos. A amostra, distribuída proporcionalmente ao número de presos por galeria e cela, foi selecionada por sorteio e incluiu detentos do regime fechado (escolha condicionada à possibilidade de acesso), que não estivessem em celas de permanência temporária (celas que abrigam, temporariamente, detentos com problemas disciplinares).

A busca ativa utilizou um instrumento de avaliação clínica que contemplava perguntas sobre a presença ou a ausência de tosse, escarro, hemoptise, febre, sudorese noturna, emagrecimento, inapetência e dispnéia. $\mathrm{O}$ instrumento foi aplicado por pesquisadores e trabalhadores do presídio que se dispuseram a contribuir na coleta de dados.

O grupo de participantes da busca passiva representa a demanda espontânea de 133 sintomáticos atendidos na unidade de saúde do presídio durante o período do estudo. Todos os detentos identificados como sintomáticos pela equipe de saúde do presídio foram incluídos, independentemente da cela e do regime de detenção.

$\mathrm{Na}$ busca passiva e ativa, casos que apresentaram tosse e escarro, por período maior ou igual a duas semanas, foram definidos como sintomáticos respiratórios e coletaram duas amostras pulmonares para exames de microscopia e cultivo. Casos com exames microbiológicos negativos em que não se descartava a possibilidade de TB, a confirmação foi realizada por diagnóstico clínico e exame radiológico de tórax ${ }^{10,11}$. As definições de casos, recomendadas a partir de 2013 pela OMS ${ }^{1}$, foram utilizadas no estudo (Quadro 1). Todos os casos tiveram seguimento clínico-terapêutico conforme estabelecido pelo Ministério da Saúde ${ }^{10}$, sendo acompanhados pelo Programa Municipal de Controle da TB e equipe de saúde prisional.

As ações de controle da TB foram desenvolvidas tendo-se como referencial teórico o Manual de Controle da TB no Brasil ${ }^{10}$. Para tanto, elaborou-se um modelo dos elementos básicos para o controle da TB em presídio (Figura 1). Nesse modelo, os contornos fechados indicam os elementos positivos do processo, as bordas tracejadas evidenciam os componentes que se apresentaram frágeis devido a problemas operacionais em sua implementação, sendo o componente sem bordas (que se refere à diminuição das taxas de incidência e prevalência de TB) aquele ainda não mensurado ou alcançado em curto prazo. 
Quadro 1. Definição de casos de TB segundo recomendações da Organização Mundial da Saúde.

\begin{tabular}{|c|c|}
\hline Caso de TB & Definição \\
\hline $\begin{array}{l}\text { Com confirmação } \\
\text { microbiológica }\end{array}$ & Paciente com amostra biológica positiva em exame de microscopia ou cultura. \\
\hline Com diagnóstico clínico & $\begin{array}{l}\text { Caso sem confirmação microbiológica, que tenha sido diagnosticado } \\
\text { clinicamente por um profissional médico. Inclui casos diagnosticados com } \\
\text { base em anormalidades no exame radiológico, histologia sugestiva ou casos de } \\
\text { TB extra-pulmonar sem confirmação microbiológica. Casos com diagnóstico } \\
\text { clínico e posterior exame microbiológico positivo (independente se antes ou } \\
\text { depois do início do tratamento) são considerados como casos com confirmação } \\
\text { microbiológica. }\end{array}$ \\
\hline Caso novo de TB & $\begin{array}{l}\text { Paciente que nunca tenha feito tratamento para TB ou que tenha usado medicações } \\
\text { por período menor que um mês. }\end{array}$ \\
\hline Caso de retratamento & $\begin{array}{l}\text { (I) Paciente anteriormente tratado que reinicia um tratamento após falha } \\
\text { terapêutica; (II) caso de paciente previamente tratado que retorna ao tratamento } \\
\text { após interrompê-lo; (III) paciente previamente declarado curado ou com } \\
\text { tratamento completo que tenha nova confirmação microbiológica de TB. }\end{array}$ \\
\hline $\begin{array}{l}\text { Multidroga-resistência } \\
\text { (MDR) }\end{array}$ & Caso em que se detecta cepa resistente a isoniazida e rifampicina. \\
\hline Cura & $\begin{array}{l}\text { Caso de TB com confirmação microbiológica no início do tratamento, que tenha } \\
\text { microscopia ou cultura negativa ao final do tratamento e em ao menos um } \\
\text { episódio anterior. }\end{array}$ \\
\hline Tratamento completo & $\begin{array}{l}\text { Paciente que completou o tratamento sem evidência de falência terapêutica, mas } \\
\text { que não possui registros de microscopia ou cultivo realizados no último mês } \\
\text { de tratamento e que teve, em pelo menos uma ocasião, exame microbiológico } \\
\text { negativo. }\end{array}$ \\
\hline $\begin{array}{l}\text { Perda para seguimento } \\
\text { clínico }\end{array}$ & $\begin{array}{l}\text { Paciente com TB que não tenha tratamento iniciado ou que tenha interrompido o } \\
\text { tratamento por dois meses ou mais. }\end{array}$ \\
\hline
\end{tabular}

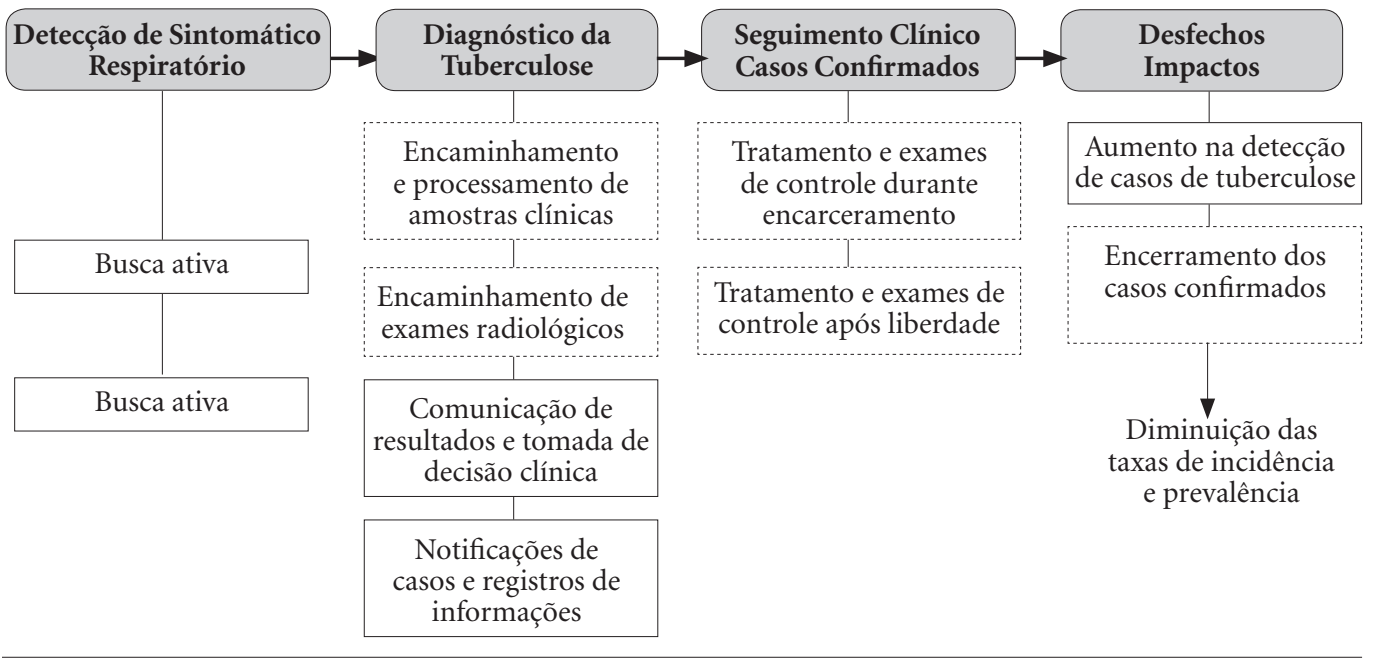

Figura 1. Elementos do processo de controle da tuberculose em presídio. 
Esse modelo também faz parte dos resultados do presente estudo.

Concomitantemente à produção de dados que permitissem estimar a magnitude da TB na população de estudo, dados qualitativos foram coletados por meio de observação participante e registros em diário de campo. A natureza participante da observação foi caracterizada devido à posição do observador frente ao grupo observado, interagindo por longos períodos com os sujeitos, buscando partilhar o cotidiano do local de estudo ${ }^{12}$ e intervindo para a implantação de medidas de controle da TB. A observação participante foi realizada nos 12 meses de busca ativa e passiva de TB, quando, duas vezes por semana, o observador permaneceu, aproximadamente, três horas por dia no interior do presídio, totalizando 288 horas de observação.

A análise dos eventos observados percorreu uma pré-análise, a exploração do diário de campo e o tratamento dos resultados ${ }^{13}$. Inicialmente, os registros foram padronizados considerandose a descrição do ambiente e dos sujeitos presentes na situação observada; o relato e a percepção do pesquisador frente ao observado. Posteriormente, foram classificados em potenciais ou em limitações para o estabelecimento de ações de controle da TB, conforme modelo da Figura 1. As informações fornecidas pela análise foram colocadas em evidência por quantificação simples.

A partir do modelo proposto e da análise por temas classificatórios descrita, os seguintes pontos para discussão do processo de detecção e tratamento da TB em presídio foram elaborados: identificação de casos de TB; avaliação clínica de presidiários mediante questionário; a fragilidade do seguimento clínico-terapêutico dos casos de TB prisional; e o envolvimento de diferentes trabalhadores e articulação com outros serviços de saúde. Este artigo é parte da tese Prevalência de tuberculose prisional: fatores de risco e epidemiologia molecular. Programa de Pós-Graduação em Enfermagem da Universidade Federal do Rio Grande (FURG), 2014. O estudo foi aprovado pelo Comitê de Ética em Pesquisa na Ârea da Saúde da Universidade Federal do Rio Grande.

\section{Resultados e discussão}

\section{Identificação de casos de tuberculose no presídio}

Através da busca ativa e passiva, foram identificados 279 detentos sintomáticos respirató- rios, 12,9\% (36/279) tiveram diagnóstico de TB confirmado (17/146 detectados por busca ativa e $19 / 133$ por busca passiva). Entre os casos, $86,11 \%$ (31/36) tiveram confirmação microbiológica de TB e $13,89 \%$ (05/36) foram diagnosticados com base em diagnóstico clínico e radiologia. Outros cinco detentos ingressaram no presídio já em tratamento para $\mathrm{TB}$, totalizando, portanto, 41 casos no período do estudo. Identificou-se como retratamento $36,58 \%(14 / 41)$ do total de casos. Embora não incluídos nesta pesquisa, destaca-se que, anteriormente ao início da coleta de dados, o local de estudo possuía 14 detentos em tratamento para TB.

A busca ativa, entendida como a implementação de ações visando identificar pessoas sintomáticas e realizar diagnóstico de TB ativa ${ }^{14}$, no presente estudo, além de permitir a detecção de sintomáticos, propiciou difundir informações sobre TB dentro do presídio. Essa afirmação está sustentada pelo observado durante pesquisa de campo, pois a mobilização de 285 detentos para avaliação por meio de busca ativa, progressivamente aumentou a demanda de busca de atendimentos pelos sintomáticos respiratórios. Fato que permitiu a constituição do grupo de busca passiva, com 133 detentos, que, estimulado pelo movimento gerado pela busca ativa, procurou a unidade de saúde do presídio para elucidar a sintomatologia respiratória.

Os detentos participantes da busca ativa foram estimulados a divulgar para os demais presos de sua convivência que todos aqueles com tosse e escarro poderiam solicitar avaliação para TB junto à equipe de saúde prisional. Sendo isso uma forma de participação/autoapresentação no processo de detecção por demanda espontânea de atendimentos, especialmente num ambiente onde a informação é restrita e seletiva.

As solicitações de avaliação para TB feitas pelos próprios presos começaram a chegar à equipe do projeto e à unidade de saúde do presídio através de bilhetes enviados das galerias. Os bilhetes eram recebidos de diferentes maneiras: $12,8 \%$ deles (17/133) por meio de trabalhadores da segurança, que durante a revista e a conferência de presos (rotina diária da primeira hora da manhã), recebiam os bilhetes e entregavam aos responsáveis pelo encaminhamento de exames; $17,3 \%(23 / 133)$ através dos detentos que trabalhavam no ambulatório, que ao fazerem a entrega de medicações de uso contínuo nas galerias, recebiam as solicitações de presos; 15/133 (11,3\%) através de assistentes sociais, dentistas e psicólogos, que ao fazerem seus atendimentos, também 
passaram a receber as mesmas solicitações. Inclusive, por vezes, sendo feitas pelo detento que estava sendo atendido, mas em nome de outro da mesma galeria (Tabela 1).

A busca passiva contribuiu para o aumento do número de casos detectados, já que entre os 133 presos sintomáticos respiratórios avaliados nesse grupo, foram encontrados 19 casos de TB, correspondendo a $14,28 \%$. Esta foi uma taxa de detecção superior à da busca ativa, 17 casos entre 146 sintomáticos $(11,64 \%)$. Entretanto, é indispensável proceder a busca ativa, porque, além de intensificar a detecção da TB, propicia a inclusão de detentos que não se percebam sintomáticos ou que não tenham a possibilidade/facilidade de acesso ao serviço de saúde do próprio presídio. Este último caso foi evidenciado neste estudo a partir de afirmações de dez entre os 285 (3,5\%) selecionados para busca ativa, de que há certo tempo colocavam seus nomes em listas de solicitação de atendimento e ainda não haviam sido chamados para avaliação.

Obstáculos à autoapresentação, como a dificuldade de acesso ao serviço de saúde, questões de gênero e fatores socioeconômicos têm sido associadas com o maior tempo entre o início dos sintomas e o diagnóstico de TB na população livre ${ }^{15,16}$.

O presente estudo não foi delineado para identificar variáveis relacionadas ao atraso no diagnóstico, mas o contexto observado sugere que os obstáculos à autoapresentação sejam de natureza diferente do que o relatado na comunidade em geral, pois, supostamente, incluam o poder e a hierarquia instituída dentro das galerias, pelos próprios presos, como parte das regras de convivência. Exemplifica-se isso ao verificar que os líderes de galeria (reconhecidos como chefes ou prefeitos) de certa forma "avaliam" a condição de saúde dos demais, pois têm a responsabilidade de repassar à segurança, uma lista diária de presos que solicitam atendimento de saúde, podendo priorizar, ou não, a inclusão de alguns. Outro es- tudo sobre saúde prisional indica que esse papel regulador possa ser exercido pelos próprios agentes de segurança, relacionando o acesso ao serviço de saúde ao bom comportamento do detento ${ }^{17}$.

Essa estrutura de poder deve ser compreendida como parte do cenário prisional e, portanto, inerente ao planejamento de saúde que venha ser implantado. Representa afirmar que o contexto prisional, em sua estrutura sócio-hierárquica, possui potencial para alterar quantitativamente o número de detentos participantes no processo de detecção e, consequentemente, terapêutico, na mesma intensidade. A qualificação de tal processo é representada pelo resultado positivo ou negativo da articulação entre busca ativa e passiva e diminuição da carga da doença nesse cenário.

\section{Avaliação clínica de presidiários mediante questionário}

Diferentes estudos indicam maior sensibilidade na detecção de casos de TB através de rastreio radiológico ${ }^{4,5,18}$. Entretanto, a disponibilidade de equipamentos e profissionais para este fim não são realidade da maioria dos presídios ${ }^{2}$. O presídio deste estudo não possui equipamentos para radiologia, assim, a mobilização de um grande número de presos até um serviço de saúde para realização de exame radiológico seria a única opção para proceder à triagem por exame de imagem, porém, este seria um obstáculo de difícil transposição, tanto pela logística, quanto pela disponibilidade de viaturas e pessoal para esse fim.

Assim, a avaliação clínica por meio de questionários tem sido método de escolha em diferentes presídios ${ }^{2}$. Neste estudo, essa também foi a opção de maior exequibilidade. Contudo, é importante descrever o quanto os avaliadores devem estar sensíveis às fragilidades impostas pelos questionários de sintomas, quando aplicados à população prisional, entre a qual, encontra-se a subvalorização dos sintomas em um ambiente

Tabela 1. Forma de recebimento da solicitação de exame de tuberculose entre casos sintomáticos respiratórios do presídio, atendidos por busca passiva.

\begin{tabular}{lcc}
\hline \multicolumn{1}{c}{$\begin{array}{c}\text { Busca passiva: forma de recebimento da solicitação } \\
\text { de exame de tuberculose }\end{array}$} & $\begin{array}{c}\text { Número de observações } \\
\text { registradas (n/N) }\end{array}$ & Total (\%) \\
\hline Através de agentes penitenciários & $17 / 133$ & $12,8 \%$ \\
Através de detentos trabalhadores da unidade de saúde & $23 / 133$ & $17,3 \%$ \\
Através de assistentes sociais, dentistas ou psicólogos & $15 / 133$ & $11,3 \%$ \\
Exames solicitados durante consulta com equipe de saúde & $78 / 133$ & $58,6 \%$ \\
\hline
\end{tabular}


violento onde a preocupação com a sobrevivência é prioritária. Sendo a aparência e a força fundamentais, a fragilidade relacionada com a doença leva a uma maior estigmatização ${ }^{17}$. Por outro lado, pode representar uma oportunidade ímpar para aqueles detentos que se sentem fragilizados física e mentalmente e que não querem expressar isso ao coletivo dos detentos e aos agentes de segurança no interior do presídio.

Observaram-se, durante a aplicação do questionário, diferentes percepções atribuídas pelos detentos para os sintomas de TB, o que pode ter interferido na triagem de casos de suspeita da doença. Embora, o instrumento não tenha sido elaborado com a finalidade de coletar informações sobre tais percepções de sintomas, na Tabela 2 os registros obtidos foram quantificados, pois expressam, em conjunto, uma significação do coletivo dos detentos a partir da autopercepção das manifestações orgânicas da doença.

Exemplificando-se tais percepções, observou-se que a ocorrência de tosse foi relatada como algo "normal", justificada por 9,2\% dos presos como consequência do tabagismo ativo ou passivo. O significado de normal pode, neste contexto, ser substituído por reflexo natural que deve ser investigado. A tosse crônica pode ser subestimada como um sintoma da doença não só no ambiente prisional, o que coloca em evidência a importância de investir esforços nas ações de educação em saúde com enfoque nos principais sinais clínicos da $\mathrm{TB}^{19}$.

Em outros 7,7\% casos, a sudorese noturna foi percebida como algo que, evidentemente, ocorria na estação do verão em uma cela pequena, sem ventilação com, em média, nove detentos em um espaço que, idealmente, abrigaria seis pessoas (existem duas celas maiores, que abrigam até 20 detentos); e o emagrecimento, a falta de apetite foram, para 3,2\% dos avaliados, apontados como resultado do encarceramento, tristeza e depres- são, ou ainda, devido aos alimentos disponíveis no presídio. Por outro lado, um eventual ganho de peso também era apontado devido à abstinência do uso de crack.

A febre pode ser outro sinal complexo de ser avaliado, pois, quando em alguns casos havia a referência a uma possibilidade de ter tido hipertermia, ela não foi confirmada pela indisponibilidade de termômetro clínico.

O ambiente físico do presídio foi mencionado por 3,9\% como a causa da falta de ar e o sentir-se debilitado. Espaços sujos, superlotados, mal iluminados e ventilados, foram ditos pelos presos como um ambiente inóspito. Tais evidências puderam ser constatadas por meio de observação participante, durante visita a uma das galerias. A relação entre as condições do ambiente físico e o adoecimento dos detentos, foi referida também em estudo qualitativo, na perspectiva dos administradores de presídios. Estes consideram que a ocorrência de doenças infecciosas esteja associada às condições das celas que, além de úmidas e com precária higiene, encontram-se superlotadas ${ }^{20}$.

Outra fragilidade na avaliação de sintomas foi a ideia de tempo no interior do presídio. Os detentos, ao serem questionados sobre o tempo em semanas para a presença de determinados sintomas, nem sempre respondiam de forma satisfatória. Para a maioria, $75,3 \%$ dos casos, isso foi descrito com precisão, da mesma maneira que respondiam sobre o tempo total de pena, em meses, semanas e dias. Mas, para o restante dos casos, a noção confusa de tempo não permitia informar, por exemplo, o período total que se encontravam na instituição ou o número de vezes que haviam sido presos. Assim, a definição operacional de detento sintomático respiratório (tosse produtiva há duas ou mais semanas) ${ }^{10}$, nem sempre pôde ser considerada com rigor na tomada de decisão para coleta de escarro.

Tabela 2. Percepções dos sintomas de tuberculose segundo detentos participantes da intervenção de busca ativa de casos.

\begin{tabular}{lcc}
\hline \multicolumn{1}{c}{ Percepções dos sintomas de TB } & $\begin{array}{c}\text { Número de observaçóes } \\
\text { registradas n/N }\end{array}$ & Total (\%) \\
\hline Presença de tosse ou escarro atribuída ao fumo & $26 / 285$ & $9,2 \%$ \\
Sudorese noturna atribuída ao calor ou falta de ventilação na cela & $22 / 285$ & $7,7 \%$ \\
Ambiente físico do presídio relacionado como causa da falta de ar e o & $11 / 285$ & $3,9 \%$ \\
sentir-se debilitado & $09 / 285$ & $3,2 \%$ \\
$\begin{array}{l}\text { Emagrecimento e falta de apetite associado a fatores emocionais ou tipo } \\
\text { de alimentação disponível }\end{array}$ & \\
\hline
\end{tabular}


Diante dessas dificuldades e visando a obtenção de melhores respostas, desdobramentos simples dos questionamentos foram necessários, tais como: verificar se a sudorese era devido ao calor (se possuíam ou não ventilador na cela); se a tosse com escarro ocorria todos os dias por muito tempo e se outros sintomas haviam aparecido além da tosse; se o emagrecimento poderia ter relação com os alimentos disponíveis e se a falta de apetite tinha relação com a tristeza. Ainda na tentativa de minimizar os fatores que impediam a adequada avaliação de sintomas, questionar também se o detento percebia-se doente e fraco foi uma estratégia importante, pois, ao mesmo tempo em que contribuiu para o entrevistador identificar a necessidade de retomar o questionamento dos sintomas, alertava o detento sobre a relação entre a TB e seus sinais clínicos. A partir disso, entre aqueles $24,7 \%$ casos que, inicialmente, não haviam respondido de forma satisfatória o questionário de avaliação, foi possível, ao menos, obter respostas que auxiliassem a decisão de encaminhar ou não exames diagnósticos. Esses aspectos podem ser considerados na elaboração de instrumentos para avaliação clínica de TB em presídios.

A existência de fragilidades das triagens por sintomas clínicos mencionadas foram constatadas, o que pode ter contribuído para uma detecção de casos abaixo da realidade. Todavia, o presente estudo mostrou taxas de prevalência e incidência alarmantes 5.366,49/100.000 (41/764) e 3.403,14/100.000 (27/764, excluídos 14 casos de retratamento), respectivamente, o que resulta numa prevalência 79 vezes superior à observada na comunidade livre do município, 68/100.000 em $2012^{21}$. Além disso, a incidência encontrada foi maior do que a taxa média dos presídios do Brasil, 1.080/100.0003.

Cabe destacar que a população flutuante do presídio durante o período de busca de casos dificulta determinar, apropriadamente, um numerador e denominador para cálculos de incidência e prevalência ${ }^{22}$. Assim, tais indicadores fornecem uma medida aproximada ou uma estimativa da magnitude da doença, não podendo ser tratados como a mensuração precisa de prevalência e incidência. Reconhecida essa limitação, tais estimativas ainda são apresentadas devido ao interesse em compará-las com outros estudos e indicadores locais de TB. Também necessita ser esclarecido que, para esses cálculos, o número de casos detectados por busca passiva e ativa foi somado, pois, em outra pesquisa realizada com a mesma população de estudo, nenhuma diferença estatisticamente significativa foi observada entre os grupos ${ }^{23}$.

As estimativas de prevalência e incidência do presente estudo, embora semelhantes ao encontrado por pesquisas em presídios da mesma região e que usaram a triagem por sintomas, seguida de diagnóstico por microscopia e cultivo ${ }^{6,24}$, são superiores a outras investigações que tiveram a radiologia como método de triagem, sendo, aqueles com imagens sugestivas de TB orientados à coleta de amostra pulmonar para microscopia e cultivo ${ }^{4,5,18,25,26}$.

Esses resultados, tanto podem estar atrelados à elevada prevalência da doença em sua forma ativa, quanto à forma como os sintomas foram avaliados neste estudo, compreendendo e tentando minimizar as limitações impostas pela avaliação clínica dentro de um presídio. Assim, considera-se a importância de que a triagem por sintomas seja feita por profissionais preparados para identificar os limites descritos e buscar formas de obter respostas adequadas à avaliação clínica de TB, não sendo eficaz, no ambiente dos presídios, utilizar-se unicamente de questionários autoaplicáveis ou concentrar esforços apenas na detecção passiva.

Além disso, é importante destacar que as estratégias de detecção por busca ativa e passiva, da forma que se desenvolveram e tendo o cultivo como ferramenta de diagnóstico laboratorial, possibilitaram aumentar quase três vezes o número de casos de TB ativa notificados: de 16 casos em 2011 para 41 casos, no período de um ano, entre 2012-13. E, mesmo com todas as limitações decorrentes da inexistência de um sistema de informação, a comunicação de resultados de exames para a tomada de decisão clínica e a notificação de casos foi efetuada adequadamente por telefone, possibilitando o início do tratamento em média $24 \mathrm{~h}$ após a confirmação diagnóstica.

Por fim, levando-se em conta os aspectos já analisados, aumentar, ainda mais, a detecção de casos neste presídio pode ser possível com a utilização de triagem por exame radiológico associada a outros métodos de diagnóstico laboratoriais. Tendo em vista que, embora tenhamos verificado uma elevada taxa de detecção realizando triagem por sintomas clínicos e avaliação por microscopia e cultivo, a radiologia como método de seleção de sintomáticos, seguido de microscopia e cultivo teve, em outro presídio, sensibilidade de $56 \%$ em relação à avaliação inicial baseada em escore clínico ${ }^{18}$. Mensurar o impacto de métodos e estratégias diagnósticas é de especial interesse em locais como os presídios. 


\section{O envolvimento de diferentes trabalhadores e a articulação com outros serviços de saúde}

O envolvimento de diferentes profissionais foi fundamental para o encaminhamento de ambas as estratégias de busca (ativa e passiva), quando, em especial, os agentes penitenciários foram os principais envolvidos. Os agentes mobilizavam os detentos até o local para entrevistas, avaliação de sintomas e orientação para coleta de escarro, quando indicado. Sem a possibilidade de mobilizar detentos, como ocorrido em situações de pouco pessoal da segurança na escala de trabalho e problemas relacionados à manutenção da disciplina no interior das galerias, não se desenvolviam as avaliações planejadas. Este é um dos exemplos da inconstância na manutenção de uma das rotinas que fazem parte do processo de controle da TB. A complexa dinâmica organizacional e funcional do presídio determina adaptações constantes, exigindo uma plasticidade comportamental em cada uma das atividades propostas, independente se na etapa de detecção, diagnóstico, seguimento clínico ou avaliação dos resultados.

Também foram identificados como importantes colaboradores neste processo os detentos trabalhadores do ambulatório da instituição, uma vez que contribuíram para organizar a demanda de atendimento da busca ativa e passiva de TB, bem como para o recolhimento das amostras coletadas e o seguimento clínico dos casos detectados. Entende-se que, em parte, esses detentos desempenharam uma posição semelhante ao dos agentes comunitários de saúde, fortalecendo o vínculo entre a equipe de saúde e os pacientes, por conhecerem o ambiente onde se inserem as práticas de saúde ${ }^{27}$.

Entre todos envolvidos no processo de controle da TB, a enfermeira aparece como a profissional da equipe de saúde que toma para si a responsabilidade de articular as ações de saúde e o pessoal da segurança. Junto à assistente social, organiza a demanda de atendimentos fora do presídio, no caso da TB, para o encaminhamento de exames laboratoriais e radiológicos, além da previsão de medicações.

Não raramente, os atrasos ou imprevistos na rotina da instituição penal, como, por exemplo, nos procedimentos envolvidos na conferência diária ou na mobilização de presos para diferentes objetivos, como as saídas para audiências, impossibilitaram o recolhimento das amostras coletadas ou levaram à perda da data agendada para realização de exame radiológico fora da instituição. Em consequência disso, havia a necessidade de nova coleta de amostras, novos agendamentos e, inevitavelmente, maior tempo de espera para a confirmação diagnóstica. Assim, se constata que a dinâmica da estrutura do presídio dificulta a inserção de outras rotinas, o que influenciou negativamente na agilidade necessária para encaminhamento de exames diagnósticos.

No contexto do estudo, os serviços de saúde envolvidos no controle da TB dentro e fora do presídio são os mesmos, o Programa Municipal de Controle da TB (PMCT), o Serviço de Assistência Especializada, o Laboratório de Análises Clínicas do município e clínicas ou hospitais para exame radiológico; articulados pela unidade de saúde do paciente, nesse caso, a de saúde prisional.

Contudo, com a restrição da autonomia sobre a própria saúde e a privação da liberdade, os presos dependem de terceiros na busca de atendimento, marcação de exames e recebimento de medicações, por exemplo. Em outro local de estudo e na perspectiva do doente apenado, o atraso no diagnóstico da TB é relacionado à naturalização da desassistência como consequência de sua condição de estar cumprindo pena privativa de liberdade ${ }^{28}$.

Ainda que no presente estudo os trabalhadores envolvidos tenham dedicado tempo e esforços na tentativa de qualificar o controle da TB, a interação entre presídio e serviços de saúde foi frágil do ponto de vista operacional. Ao menos, em parte, isso se deve ao fato desta articulação ter dependido, quase que exclusivamente, de pessoas que compreendiam a TB no presídio como um problema. Como consequência, maior parte das ações centralizaram-se naqueles trabalhadores sensibilizados com isso. De tal forma, o eventual afastamento dessas pessoas poderia determinar a descontinuidade das estratégias de controle da TB desenvolvidas. Logo, há a necessidade de implementar todas as ações como um processo, tomando, como base, um algoritmo adequado às especificidades do presídio, com rotinas conhecidas e estabelecidas tanto pelos serviços de saúde, quanto pelo presídio, não apenas pelos indivíduos envolvidos em um determinado momento.

\section{As fragilidades do seguimento clínico-terapêutico dos casos de TB em presídio}

O aparente controle da rotina dos detentos dentro presídio transmite a ideia de facilidade 
para o seguimento clínico dos casos de TB que, no modelo elaborado para este estudo, considera o tratamento e os exames de controle durante o encarceramento e após a liberdade. Entretanto, diferentes situações evidenciaram o contrário, ou seja, a condição de reclusão dificulta a supervisão clínica-terapêutica do doente.

A primeira está relacionada ao momento que o preso em tratamento recebe liberdade dificultando uma abordagem para orientar sobre o seguimento clínico fora do presídio. Não existe um controle exato sobre a data de recebimento de um alvará de soltura, além disso, por vezes o detento tem liberdade em período que não há pessoal de saúde disponível. O que melhor reflete isso ocorreu com um caso de TB causada por cepa multidroga-resistente. Esse caso, com história de tratamento irregular dentro do presídio e abandono de tratamentos anteriores foi, novamente perdido para o seguimento clínicoterapêutico, uma vez que, após receber liberdade houve descontinuidade do tratamento e não foi possível localizar o doente. A previsão de liberdade foi informada pelo próprio preso, em uma das consultas de acompanhamento do tratamento (ainda não se sabia o perfil de sensibilidade aos antibióticos do microrganismo). Embora orientado acerca do tratamento fora do presídio, por ser usuário de crack, o próprio doente previa não seguir a tomada de medicação após liberdade. A partir do endereço informado não foi possível localizá-lo. Cinco meses depois, observou-se registro de entrada desse mesmo caso no Pronto Socorro Municipal e, no momento de permanência na unidade, a equipe do local desconhecia o histórico de TB do paciente. Assim, devido à inexistência de um sistema de informação, novamente perdeu-se a oportunidade de seguimento clínico. Isso, portanto, não pode unicamente ser atribuído a fragilidade do presídio em questão, mas do contexto onde a unidade penal se insere.

Verificou-se no PMCT que o paciente em questão segue sem tratamento, o que representa, além de um risco individual, a possibilidade da transmissão de cepa resistente e a ocorrência de casos de resistência primária, até agora não registrados no município.

Em outras quatro situações, os detentos com TB que receberam liberdade buscaram atendimento no PMCT, entretanto, referiram não ter recebido a medicação regularmente no presídio. Devido à sobrecarga de trabalho e à pouca disponibilidade de trabalhadores da saúde, não foi viável observar a tomada diária de medicação conforme recomendações do Programa Nacional de
Controle da $\mathrm{TB}^{4}$. Com isso, as medicações eram entregues mensalmente, os detentos assinavam uma lista de recebimento e, prioritariamente, eram avaliados pela equipe no segundo, quarto e sexto mês de tratamento, quando também se realizava o encaminhamento de exames de controle. Os casos que relataram a entrega irregular da medicação possuem suas assinaturas nos registros de controle de recebimento, contudo, referiram a possibilidade de que outras pessoas tenham feito o recebimento da medicação.

Tal possibilidade pode ser considerada se avaliarmos que as condições de atendimento ao doente de TB no presídio eram, em determinadas circunstâncias, vistas como um benefício. Primeiro, pelo recebimento de leite, não incluso na alimentação de quem não tenha diagnóstico de TB, segundo, por três situações observadas sugerirem que os medicamentos tenham sido elementos de troca no presídio. Esse entendimento de que estar com TB no presídio seja, eventualmente, algo positivo, pode também influenciar a adesão ao tratamento e, consequentemente, a cura, na tentativa de manter benefícios ${ }^{29}$.

Em outro presídio da mesma região, 96\% dos pacientes relataram a tomada irregular de medicação ${ }^{6}$. No sistema prisional de São Paulo, a análise de dados retrospectivos do período de sete anos indica que a taxa de abandono chegou a $49,9 \% \%^{30}$. Relacionando os dados da presente pesquisa, verifica-se que em 24,4\% (10/41) houve perda para seguimento clínico-terapêutico (04 relataram receber irregularmente a medicação no presídio, 02 interromperam tratamento após liberdade, 02 não iniciaram o tratamento por terem tido liberdade e 02 interromperam o tratamento dentro do presídio).

Dois detentos foram diagnosticados com TB causada por cepa resistente $(7,79 \%)$, sendo um caso simultaneamente resistente à isoniazida e rifampicina e outro à isoniazida e etambutol. Ambos, casos de retratamento, um com seguimento clínico de tratamento atual descontinuado (conforme anteriormente relatado) e outro curado, após devido encaminhamento para avaliação no centro de referência, embora todas as dificuldades de logística para isso, pois tal centro se localiza a mais de $250 \mathrm{~km}$ do presídio. A taxa de resistência relatada neste estudo é semelhante à média descrita por outro estudo sobre TB prisional, 6,2\% dos isolados clínicos resistentes a pelo menos um antibiótico ${ }^{30}$.

Apesar desse indicador negativo, destaca-se que $75,6 \%$ (31/41) do total de casos tiveram desfecho positivo (cura em 23/41 e tratamento com- 
pleto em 8/41 casos). Mas, segundo a nova definição de cura proposta pela OMS (Quadro 1), apenas 56\% (23/41) dos detentos com TB foram curados, muito aquém da taxa mínima de cura preconizada, $85 \%^{1}$.

\section{Conclusões}

A busca ativa, como um instrumento para aumentar a detecção passiva de casos, a adequada comunicação de resultados laboratoriais visando o início imediato do tratamento e o aumento no número de casos diagnosticados, foram potencialidades do processo de controle da TB encontrados no cenário deste estudo.

A avaliação clínica por meio de questionário contribuiu para triplicar a detecção de casos de TB. Resultado que dependeu tanto do reconhecimento das fragilidades desse tipo de triagem, quanto do envolvimento ativo de agentes de segurança, equipe de saúde, assistentes sociais e psicólogos.

As dificuldades encontradas para a implementação do processo de controle da TB estiveram atreladas à interação entre presídio e outros serviços de saúde, em especial para a realização de exames e seguimento clínico dentro e fora do presídio, o que, inevitavelmente, impactou negativamente no encerramento de casos.

A inexistência de estudos semelhantes a este coloca em evidência esta pesquisa, mas pode, por outro lado, ter limitado a discussão dos resultados encontrados. Considera-se que os benefícios individuais e coletivos, intra e extramuros, da implementação de ações de controle da TB nos presídios sejam previsíveis. Contudo, devem ser sustentados por estudos longitudinais que permitam não somente avaliar o impacto de estratégias de controle sobre a incidência, a prevalência e as taxas de TB causada por cepas resistentes, como, também, identificar as melhores intervenções para deteç̧ão e acompanhamento clínicoterapêutico.

Este estudo fornece informações úteis à formulação de mudanças necessárias para o controle da TB no ambiente prisional. Confirma-se o pressuposto de que o controle da TB, quando abordado pela detecção e tratamento de casos, tenha o planejamento de suas ações contextualizado às particularidades inerentes a um ambiente prisional específico, bem como, à rede de serviços e trabalhadores envolvidos no controle e pesquisa da TB em cada município e/ou macrorregião. 


\section{Colaboradores}

MS Valença trabalhou na concepção e delineamento da pesquisa, na produção, análise e interpretação dos dados e na redação do artigo; MR Cezar-Vaz contribuiu para a revisão crítica de conteúdo do artigo; CB Brum participou da metodologia e produção de dados e PEA Silva trabalhou interpretação dos dados, na revisão crítica e aprovação da versão a ser publicada.

\section{Agradecimentos}

Os autores agradecem aos trabalhadores do Programa Municipal de Controle da Tuberculose, do Laboratório Municipal de Análises Clínicas e do Presídio Regional, em especial aos agentes de segurança, equipe de saúde e equipe técnica, por todo o apoio necessário no desenvolvimento da pesquisa.

\section{Referências}

1. World Health Organization (WHO). Global Tuberculosis Report 2014. Geneva: WHO; 2014.

2. Vinkeles Melchers NVS, van Elsland SL, Lange JMA, Borgdorff MW, van den Hombergh J. State of Affairs of Tuberculosis in Prison Facilities: A Systematic Review of Screening Practices and Recommendations for Best TB Control. PLoS ONE 2013; 8(1):e53644.

3. Brasil. Ministério da Saúde (MS). Secretaria de Vigilância em Saúde, Programa Nacional de Controle da Tuberculose. Situação da Tuberculose no Brasil. Brasília: MS; 2014.

4. Sánchez AR, Gerhardt G, Natal S, Capone D, Espinola AB, Costa W, Pires J, Barreto A, Biondi E, Larouzé B. Prevalence of pulmonary tuberculosis and comparative evaluation of screening strategies in a Brazilian prison. Int J Tuberc Lung Dis 2005; 9(6):633-639.

5. Sánchez AR, Larouzé B, Espinola AB, Pires J, Capone D, Gerhardt G, Cesconi V, Procopio MJ, Hijjar M, Massari V. Screening for tuberculosis on admission to highly endemic prisons? The case of Rio de Janeiro State prisons. Int J Tuberc Lung Dis 2009; 13(10):1247-1252.

6. Rocha JZ, Valença MS, Carrion LL, Silva LV, von Groll A, Silva PA. Respiratory symptoms and active tuberculosis in a prison in Southern Brazil: Associated epidemiologic variables. Rev Epidemiol Control Infect 2013; 3(4):128-133.

7. World Health Organization (WHO). Prisons and Health. Geneva: WHO; 2014.

8. Driessnack M, Sousa VD, Mendes IAC. Revisão dos desenhos de pesquisa relevantes para enfermagem: Parte 3: Métodos mistos e múltiplos. Rev Latino-Am Enfermagem 2007; 15(5):1046-1049.

9. Sánchez AR, Massari V, Gerhardt G, Barreto AW, Cesconi V, Pires J. Tuberculosis in Rio de Janeiro prisons, Brazil: an urgent public health problem. Cad Saude Publica 2007; 23(3):545-552.

10. Brasil. Ministério da Saúde (MS). Secretaria de Vigilância em Saúde. Departamento de Vigilância Epidemiológica. Manual de recomendações para o controle da tuberculose no Brasil. Brasília: MS; 2011.

11. Brasil. Ministério da Saúde (MS). Secretaria de Vigilância em Saúde. Programa Nacional de Controle da Tuberculose. Manual nacional de vigilância laboratorial da tuberculose e outras Micobactérias. Brasília: MS; 2008.

12. Queiroz DT, Vall J, Souza AMA, Vieira NFC. Observação Participante na Pesquisa Qualitativa: Conceitos e Aplicações na Área da Saúde. R Enferm UERJ 2007; 15(2):276-283.

13. Bardin L. Análise de conteúdo. Lisboa: Edições 70; 1977.

14. Golub JE, Mohan CI, Comstock GW, Chaisson RE. Active case finding of tuberculosis: historical perspective and future prospects. Int J Tuberc Lung Dis 2005; 9(11):1183-1203.

15. Needham DM, Foster SD, Tomlinson G, Godfrey-Faussett P. Socio-economic, gender and health services factors affecting diagnostic delay for tuberculosis patients in urban Zambia. Trop Med Int Health 2001; 6(4):256-259.

16. Yang W, Gounder CR, Akande T, Neve J, McIntire KN, Chandrasekhar A, Pereira AL, Gummadi N, Samanta A, Gupta A. Barriers and Delays in Tuberculosis Diagnosis and Treatment Services: Does Gender Matter? Tuberc Res Treat 2014; 2014:461935. 
17. Diuana V, Lhuilier D, Sanchez AR, Araújo L, Duarte AM, Garcia M, Milanez E, Poubel L, Romano E, Larouzé B. Saúde em prisões: representações e práticas dos agentes de segurança penitenciária no Rio de Janeiro, Brasil. Cad Saude Publica 2008; 24(8):1887-1896.

18. Fournet N, Sanchez A, Massari V, Penna L, Natal S, Biondi E, Larouzé B. Development and evaluation of tuberculosis screening scores in Brazilian prisons. Public Health 2006; 120(10):976-983.

19. Machado ACFT, Steffen RE, Oxlade O, Menzies D, Kritski A, Trajman A. Factors associated with delayed diagnosis of pulmonary tuberculosis in the state of Rio de Janeiro, Brazil. J Bras Pneumol 2011; 37(4):512-520.

20. Fernando Balvedi Damas. Assistência e condições de saúde nas prisões de Santa Catarina, Brasil. Rev Saude Publ 2012; 5(3):6-22.

21. Brasil. Ministério da Saúde (MS). Secretaria de Vigilância em Saúde. Sistema de Informação de Agravos de Notificação. Tuberculose - casos notificados no Sistema de Informação de Agravos de Notificação 2012. Sinan Net. [acessado 2013 out 18]. Disponível em: http://dtr 2004.saude.gov.br/sinsnweb

22. Rieder HL, Anderson C, Dara M, Hauer B, Helbling P, Kam KM, Zwahlen M. Methodological issues in quantifying the magnitude of the tuberculosis problem in a prison population. Int J Tuberc Lung Dis 2011; 15(5):662-667.

23. Valença MS, Scaini JL, Abilleira F, Gonçalves C, Von Groll A, Silva PEA. High prevalence of tuberculosis in prison: risk factors and molecular epidemiology. Int $J$ Tuberc Lung Dis 2015; 19(10):1182-1187.

24. Kühleis D, Ribeiro AW, Costa ERD, Cafrune PI, Schmid KB, Costa LL, Ribeiro MO, Zaha A, Rossetti MLR. Tuberculosis in a southern Brazilian prison. Mem Inst Oswaldo Cruz 2012; 107(7):909-915.

25. Telisinghe L, Fielding KL, Malden JL, Hanifa Y, Churchyard GJ, Grant AD, Charalambous S. High Tuberculosis Prevalence in a South African Prison: The Need for Routine Tuberculosis Screening. PLoS ONE 2014; 9(1):e87262.
26. Winetsky DE, Almukhamedov O, Pulatov D, Vezhnina N, Dooronbekova A, Zhussupov B. Prevalence, Risk Factors and Social Context of Active Pulmonary Tuberculosis among Prison Inmates in Tajikistan. PLoS ONE 2014; 9(1):e86046.

27. Brasil. Ministério da Saúde (MS). Secretaria de Atenção à Saúde. Departamento de Atenção Básica. O trabalho do agente comunitário de saúde. Brasília: MS; 2009.

28. Souza KMJ, Villa TCS, Assolini FEP, Beraldo AA, França UM, Protti ST, Palha PF. Atraso no diagnóstico da tuberculose em sistema prisional: a experiência do doente apenado. Text Context Nursing 2012; 21(1):17-25.

29. Drobniewski FA, Balabanova YM, Ruddy MC, Graha MC, Kuznetzov SI, Gusarova GI, Zakharova SM, Melentyev AS, Fedorin IM. Tuberculosis, HIV seroprevalence and intravenous drug abuse in prisioners. Eur Respir J 2005; (26):298-304.

30. Pedro HSP, Nardi SMT, Pereira MIF, Goloni MRA, Pires FC, Tolentino FM, Oliveira RS, Rossit ARB. Mycobacterium tuberculosis detection in the penitentiary system. Rev Patol Trop 2011; 40(4):287-295.

Artigo apresentado em 18/03/2015

Aprovado em 31/07/2015

Versão final apresentada em 02/08/2015 